\title{
Correlation-based Algorithm for Multi-Dimensional Single-Tone Frequency Estimation
}

\author{
Weize Sun, H.C. So and Lanxin Lin \\ Department of Electronic Engineering, City University of Hong Kong
}

\begin{abstract}
In this paper, parameter estimation for a $R$-dimensional $(R$-D) single cisoid with $R \geq 2$ in additive white Gaussian noise is addressed. By exploiting the correlation of the data samples, we construct $R$ single-tone sequences which contain the $R$-D frequency parameters. Based on linear prediction and weighted linear squares techniques, two proposals are developed for fast and accurate frequency estimation from each constructed sequence. The two devised estimators are proved to be asymptotically unbiased while their variances achieve Cramér-Rao lower bound when the signal-to-noise ratio and/or data length tend to infinity. Computer simulations are also included to compare the proposed approach with conventional $R$-D harmonic retrieval schemes in terms of mean square error performance and computational complexity.
\end{abstract}

Keywords: frequency estimation, multi-dimensional spectral analysis, fast algorithm, correlation

\section{Introduction}

Estimating the parameters of sinusoidal components from a finite number of noisy discrete-time measurements has been an important research topic be- 
cause it corresponds to many science and engineering problems [1]-[3]. Apart from the standard one-dimensional signal model [4]-[6], multi-dimensional spectral estimation [7] in fact has many applications such as array processing [8]-[9], nuclear magnetic resonance (NMR) spectroscopy [10], wireless communication channel estimation [11]-[12] as well as detection and localization of multiple targets using multiple-input multiple-output (MIMO) radar [13].

The crucial step in $R$-dimensional ( $R$-D) sinusoidal parameter estimation, with $R \geq 2$, is to find the frequency parameters which are nonlinear in the observed data. For 2-D frequency estimation, maximum-likelihood (ML) [14][15] and subspace [8]-[13], [16]-[17] approaches are commonly used choices. For higher dimensional signals, the latter seems to be the only practical solution although its computational requirement is very high. State-of-the-art subspace methods include MUSIC [9], decoupled root-MUSIC [17], unitary ESPRIT (UE) [8], rank reduction estimator (RARE) [12], multi-dimensional folding (MDF) [11] and improved MDF (IMDF) [16], and they usually need eigenvalue decomposition (EVD) or singular value decomposition (SVD) in the algorithm implementation. By effectively utilizing the correlation of the observed data, we contribute to devising an accurate frequency estimator for a $R$-D single-tone with less computational load in this work.

The rest of the paper is organized as follows. To facilitate the understanding of our underlying idea, we first develop the correlation-based approach for a 2-D cisoid in the presence of white Gaussian noise in Section 2. By exploiting correlation on the 2-D raw data, a novel single-tone sequence whose frequency corresponds to one of the two dimensions is constructed. The generalized weighted linear predictor (GWLP) [18], which can provide optimum 
performance for 1-D single-tone in white noise, is then applied for estimation of the two frequency parameters in a separable manner. Note that linear prediction (LP) and weighted least squares (WLS) are two key ingredients in the GWLP. Two estimators are devised, and their mean and variance expressions are produced. It is worthy to point out that the derivation of the weighting matrix and performance analysis are different from those of [18]. Section 3 generalizes the proposed solutions to higher dimensional signals with the use of tensor algebra [19]. Simulation results are included in Section 4 to corroborate the theoretical development and to compare the correlation-based approach with the approximate iterative quadratic ML (AIQML) [15], IMDF [16] and UE [8] algorithms as well as Cramér-Rao lower bound (CRLB) [20]. It is demonstrated that the proposed methods are more computationally efficient and their performance is close to CRLB particularly when the signalto-noise ratio (SNR) is sufficiently high. Finally, conclusions are drawn in Section 5.

\section{Two-dimensional Frequency Estimation}

We first define the notation as follows. Scalars, vectors, matrices and tensors are denoted by italic, bold lower-case, bold upper-case and bold calligraphic symbols, respectively. The magnitude, angle, real and imaginary parts of a complex scalar $a$ are $|a|, \angle(a), \Re\{a\}$ and $\Im\{a\}$, while the variable, noise-free value and estimate of a vector $\mathbf{a}$ are represented by $\tilde{\mathbf{a}}, \overline{\mathbf{a}}$ and $\hat{\mathbf{a}}$, respectively. The mean and variance of $\hat{a}$ are denoted by $\mathbb{E}\{\hat{a}\}$ and $\operatorname{var}(\hat{a})$. The $\mathbf{I}_{i}$ is the $i \times i$ identity matrix and $\mathbf{0}_{i \times j}$ is the $i \times j$ zero matrix. The operators for transpose, complex conjugate, conjugate transpose, inverse and 
Khatri-Rao product $\operatorname{are}^{T},{ }^{*},{ }^{H},{ }^{-1},{ }^{\dagger}$ and $\odot$, respectively. The $m$ th element of $\mathbf{a} \in \mathbb{C}^{M \times 1}$ and $(m, n)$ entry of $\mathbf{A} \in \mathbb{C}^{M \times N}$ are denoted by $[\mathbf{a}]_{m}$ and $[\mathbf{A}]_{m, n}$, respectively, while we use $a_{m_{1}, m_{2}, \ldots, m_{R}}$ to represent the $\left(m_{1}, m_{2}, \ldots, m_{R}\right)$ entry of a $R$-D tensor $\mathcal{A} \in \mathbb{C}^{M_{1} \times M_{2} \times \cdots \times M_{R}}$ where $R \geq 3$.

In matrix form, the 2-D signal model with size $M_{1} \times M_{2}$ is expressed as:

$$
\mathbf{Y}=\mathbf{X}+\mathbf{Q}
$$

where

$$
[\mathbf{X}]_{m_{1}, m_{2}}=\gamma e^{j\left(m_{1} \omega_{1}+m_{2} \omega_{2}\right)}, \quad m_{r}=1,2, \cdots, M_{r}, \quad r=1,2
$$

is the noise-free tone sample, and $[\mathbf{Q}]_{m_{1}, m_{2}}$ is a zero-mean complex white Gaussian process with unknown variance $\sigma_{Q}^{2}$. Moreover, $\gamma$ is the complex amplitude while $\omega_{1} \in(-\pi, \pi)$ and $\omega_{2} \in(-\pi, \pi)$ are the frequencies, and they are all unknown constants. Here, the task is to determine $\omega_{1}$ and $\omega_{2}$ from the observed matrix $\mathbf{Y}$ with $M=M_{1} M_{2}$ samples.

The main idea of the algorithm development is to exploit correlation to convert $\mathbf{Y}$ into a vector which contains the information of $\omega_{1}$ or $\omega_{2}$. That is, $\omega_{1}$ and $\omega_{2}$ are estimated in a separable manner. We notice that $\mathbf{X}$ is of rank 1 and can be factorized as

$$
\mathbf{X}=\gamma \mathbf{g}_{1} \mathbf{g}_{2}^{T}
$$

where

$$
\mathbf{g}_{r}=\left[\begin{array}{llll}
e^{j \omega_{r}} & e^{j 2 \omega_{r}} & \cdots & e^{j M_{r} \omega_{r}}
\end{array}\right]^{T}, \quad r=1,2
$$

Defining $\mathbf{X}=\left[\begin{array}{llll}\mathbf{x}_{1} & \mathbf{x}_{2} & \cdots & \mathbf{x}_{M_{2}}\end{array}\right]$ and writing $\mathbf{Y}$ and $\mathbf{Q}$ in the same way, we easily obtain the LP property:

$$
\begin{gathered}
\mathbf{x}_{1}=e^{j \omega_{2}} \gamma \mathbf{g}_{1} \\
\mathbf{x}_{m}=e^{j \omega_{2}} \mathbf{x}_{m-1}
\end{gathered}
$$


As a result, pre-multiplying $\mathbf{X}$ by $\mathbf{x}_{1}^{H}$ gives $|\gamma|^{2} M_{1} e^{-j \omega_{2}} \mathbf{g}_{2}^{T}$, which is a singletone vector with frequency $\omega_{2}$. Since $\mathbf{X}$ is not available, we use its noisy version which is given by the zero-lag correlation between all columns of $\mathbf{Y}$ and its first column vector to achieve frequency estimation:

$$
\mathbf{z}_{(2)}^{T}=\mathbf{y}_{1}^{H} \mathbf{Y}
$$

with elements:

$$
\left[\mathbf{z}_{(2)}\right]_{m_{2}}=\mathbf{y}_{1}^{H} \mathbf{y}_{m_{2}}=\mathbf{y}_{1}^{H}\left(\mathbf{x}_{m_{2}}+\mathbf{q}_{m_{2}}\right)
$$

Note that the subscript of ${ }_{(2)}$ in $\mathbf{z}$ refers to $\omega_{2}$. According to the LP property, we obtain $\mathbf{y}_{1}^{H} \mathbf{x}_{m}=e^{j \omega_{2}} \mathbf{y}_{1}^{H} \mathbf{x}_{m-1}$. Hence (8) satisfies:

$$
\left[\mathbf{z}_{(2)}\right]_{m_{2}}-e^{j \omega_{2}}\left[\mathbf{z}_{(2)}\right]_{m_{2}-1}=\mathbf{y}_{1}^{H}\left(\mathbf{q}_{m_{2}}-e^{j \omega_{2}} \mathbf{q}_{m_{2}-1}\right), \quad m_{2}=2,3, \cdots, M_{2}
$$

Denoting $\mathbf{z}_{(2) 1}=\left[\begin{array}{llll}{\left[\mathbf{z}_{(2)}\right]_{1}} & {\left[\mathbf{z}_{(2)}\right]_{2}} & \cdots & {\left[\mathbf{z}_{(2)}\right]_{M_{2}-1}}\end{array}\right]^{T}$ and $\left.\mathbf{z}_{(2) 2}=\left[\begin{array}{llll}{\left[\mathbf{z}_{(2)}\right]_{2}} & {\left[\mathbf{z}_{(2)}\right]_{3}} & \cdots & {\left[\mathbf{z}_{(2)}\right.}\end{array}\right]_{M_{2}}\right]^{T}$ and assigning $\rho_{2}=e^{j \omega_{2}}$, we construct the LP error vector based on (9):

$$
\mathbf{e}_{(2)}=\mathbf{z}_{(2) 2}-\rho_{2} \mathbf{z}_{(2) 1}
$$

The $\omega_{2}$ is then estimated by minimizing the following WLS cost function:

$$
J\left(\tilde{\rho}_{2}\right)=\left(\mathbf{z}_{(2) 2}-\tilde{\rho}_{2} \mathbf{z}_{(2) 1}\right)^{H} \mathbf{W}_{M_{2}-1}\left(\mathbf{z}_{(2) 2}-\tilde{\rho}_{2} \mathbf{z}_{(2) 1}\right)
$$

where $\mathbf{W}_{M_{2}-1} \in \mathbb{C}^{\left(M_{2}-1\right) \times\left(M_{2}-1\right)}$ is a weighting matrix. Differentiating $J\left(\tilde{\rho}_{2}\right)$ with respect to $\tilde{\rho}_{2}$ and then setting the resultant expression to zero, we get the estimate of $\rho_{2}$ :

$$
\hat{\rho}_{2}=\frac{\mathbf{z}_{(2) 1}^{H} \mathbf{W}_{M_{2}-1} \mathbf{z}_{(2) 2}}{\mathbf{z}_{(2) 1}^{H} \mathbf{W}_{M_{2}-1} \mathbf{z}_{(2) 1}}
$$


In Appendix A, we have derived the optimal weighting matrix, denoted by $\mathbf{W}_{M_{2}-1}\left(\omega_{2}\right)$, which is a function of the unknown frequency $\omega_{2}$ :

$$
\mathbf{W}_{M_{2}-1}\left(\omega_{2}\right)=\left[\mathbb{E}\left\{\mathbf{e}_{(2)} \mathbf{e}_{(2)}^{H}\right\}\right]^{-1}=\mathbf{P}^{-1}\left(\omega_{2}\right)
$$

where

$$
\mathbf{P}\left(\omega_{2}\right)=\left[\begin{array}{cccccc}
2+\left(M / M_{2}-1\right) /(\mathrm{SNR}+1) & -e^{j \omega_{2}} & 0 & 0 & \cdots & 0 \\
-e^{-j \omega_{2}} & 2 & -e^{j \omega_{2}} & 0 & \cdots & 0 \\
\vdots & \vdots & \vdots & \vdots & \vdots & \vdots \\
0 & \cdots & 0 & -e^{-j \omega_{2}} & 2 & -e^{j \omega_{2}} \\
0 & 0 & \cdots & 0 & -e^{-j \omega_{2}} & 2
\end{array}\right]
$$

with $\mathrm{SNR}=|\gamma|^{2} / \sigma_{Q}^{2}$.

In the following, two iterative algorithms are proposed to approximate the conceptual solution of (12). As SNR is unknown, our first proposal is to set $\left[\mathbf{P}\left(\omega_{2}\right)\right]_{1,1}=2$ which is valid particularly when SNR is sufficiently large, namely, SNR $>>M / M_{2}$. Under this approximation, $\mathbf{P}\left(\omega_{2}\right)$ has a closed-form inverse and the phase of $\mathbf{z}_{(2) 1}^{H} \mathbf{W}_{M_{2}-1}\left(\omega_{2}\right) \mathbf{z}_{(2) 1}$ in (12) is zero [18]. Employing the substitution of $\hat{\rho}_{2}=e^{j \hat{\omega}_{2}},(12)$ is simplified to

$$
\hat{\omega}_{2}=\angle\left(\mathbf{z}_{(2) 1}^{H} \mathbf{W}_{M_{2}-1}\left(\omega_{2}\right) \mathbf{z}_{(2) 2}\right)
$$

where the elements of $\mathbf{W}_{M_{2}-1}\left(\omega_{2}\right)$ are:

$$
\begin{aligned}
{\left[\mathbf{W}_{M_{2}-1}\left(\omega_{2}\right)\right]_{m, n} } & =\frac{M_{2} \min (m, n)-m n}{M_{2}} e^{j(m-n) \omega_{2}} \\
m & =1,2, \cdots, M_{2}-1, \quad n=1,2, \cdots, M_{2}-1
\end{aligned}
$$


In this study, we apply the GWLP [18] algorithm which updates $\hat{\omega}_{2}$ and $\mathbf{W}_{M_{2}-1}\left(\omega_{2}\right)$ in an iterative manner. We refer this estimator to as correlation1 (C-1) method. The steps of finding $\hat{\omega}_{2}$ are summarized in Table 1. The estimation of $\hat{\omega}_{1}$ is performed using the same procedure on $\mathbf{Y}^{T}$.

(i) Compute the $\mathbf{z}_{(2)}$ using (7)

(ii) Obtain an initial value of $\hat{\omega}_{2}$ using (15) with $\left[\mathbf{W}_{M_{2}-1}\left(\omega_{2}\right)\right]_{m, n}=0$ for $m \neq n$ in (16), which is in fact the weighted linear predictor estimate [21]

(iii) Construct $\mathbf{W}_{M_{2}-1}\left(\omega_{2}\right)$ according to (16) with $\omega_{2}=\hat{\omega}_{2}$

(iv) Compute an updated $\hat{\omega}_{2}$ using (15)

(v) Repeat Steps (iii)-(iv) until a stopping criterion is reached. In this study, we stop for $\tau$ iterations.

Table 1: Estimation algorithm for $\omega_{2}$

In Appendix B, we have shown that when the frequency estimation error is sufficiently small, the mean and variance of $\hat{\omega}_{r}, r=1,2$, are:

$$
\begin{aligned}
\mathbb{E}\left\{\hat{\omega}_{r}\right\} & \approx \omega_{r} \\
\operatorname{var}\left(\hat{\omega}_{r}\right) & \approx \frac{6\left(|\gamma|^{2}+\sigma_{Q}^{2}\right) \sigma_{Q}^{2}}{\left(M / M_{r}|\gamma|^{2}+\sigma_{Q}^{2}\right) M_{r}\left(M_{r}^{2}-1\right)|\gamma|^{2}}
\end{aligned}
$$

Equation (17) means that the algorithm is an asymptotically unbiased estimator. The CRLB for $\omega_{r}$, denoted by $\operatorname{CRLB}\left(\omega_{r}\right)$, is [20]:

$$
\operatorname{CRLB}\left(\omega_{r}\right)=\frac{6 \sigma_{Q}^{2}}{M\left(M_{r}^{2}-1\right)|\gamma|^{2}}
$$


To study the limit of the C-1 estimator, we define the efficiency, which is the ratio of CRLB to the variance, denoted by:

$$
\operatorname{eff}_{1}\left(\omega_{r}\right)=\frac{\operatorname{CRLB}\left(\omega_{r}\right)}{\operatorname{var}\left(\hat{\omega}_{r}\right)} \approx \frac{\left(|\gamma|^{2}+M_{r} / M \sigma_{Q}^{2}\right)}{\left(|\gamma|^{2}+\sigma_{Q}^{2}\right)}=\frac{\left(\mathrm{SNR}+M_{r} / M\right)}{(\mathrm{SNR}+1)}
$$

whose value is between 0 and 1 . It is easy to see that the C-1 method achieves optimum performance when $\mathrm{SNR} \rightarrow \infty$, with $\operatorname{eff}_{1}\left(\omega_{r}\right)=1$.

On the other hand, the above development may not be appropriate when $M / M_{r}, r=1,2$, is large particularly when $\mathrm{SNR}>>M / M_{r}$ does not hold. To tackle this scenario, our second approximation on (12) is to discard $\left[\mathbf{z}_{(2)}\right]_{1}$ of (7) in the computation. Let $\mathbf{z}_{(r) s}$ be $\mathbf{z}_{(r)}$ without the first element and follow (9)-(16), we obtain

$$
\hat{\omega}_{r}=\angle\left(\mathbf{z}_{(r) s 1}^{H} \mathbf{W}_{M_{r}-2}\left(\omega_{r}\right) \mathbf{z}_{(r) s 2}\right)
$$

where

$$
\begin{aligned}
& \mathbf{z}_{(r) s 1}=\left[\begin{array}{llll}
{\left[\mathbf{z}_{(r) s}\right]_{1}} & {\left[\mathbf{z}_{(r) s}\right]_{2}} & \cdots & {\left[\mathbf{z}_{(r) s}\right]_{M_{r}-2}}
\end{array}\right]^{T} \\
& \mathbf{z}_{(r) s 2}=\left[\begin{array}{llll}
{\left[\mathbf{z}_{(r) s}\right]_{2}} & {\left[\mathbf{z}_{(r) s}\right]_{3}} & \cdots & {\left[\mathbf{z}_{(r) s}\right]_{M_{r}-1}}
\end{array}\right]^{T}
\end{aligned}
$$

We refer this estimator to as correlation-2 (C-2) method. As a result, the estimate of (21) is obtained in a similar manner as in Table 1.

Following Appendix A and replacing $M_{r}$ by $M_{r}-1$, we see that the frequency estimate is also asymptotically unbiased and its variance is:

$$
\operatorname{var}\left(\hat{\omega}_{r}\right) \approx \frac{6\left(|\gamma|^{2}+\sigma_{Q}^{2}\right) \sigma_{Q}^{2}}{\left(M / M_{r}|\gamma|^{2}+\sigma_{Q}^{2}\right)\left(M_{r}-1\right)\left[\left(M_{r}-1\right)^{2}-1\right]|\gamma|^{2}}
$$

Analogous to (20), the efficiency of this estimator is

$$
\operatorname{eff}_{2}\left(\omega_{r}\right)=\frac{\left(M_{r}-1\right)\left[\left(M_{r}-1\right)^{2}-1\right]\left(\mathrm{SNR}+M_{r} / M\right)}{M_{r}\left(M_{r}^{2}-1\right)(\mathrm{SNR}+1)} \in(0,1)
$$

and we see that $\operatorname{eff}_{2}\left(\omega_{r}\right)=1$ when both SNR and $M_{r}$ tend to infinity. 


\section{Extension to Higher Dimension}

In this section, we generalize our development to higher dimensional signals with $R \geq 3$, where tensor representation is employed. The observed $R$-D sinusoidal signal is modeled as:

$$
\mathcal{Y}=\mathcal{X}+\mathcal{Q}
$$

where

$$
x_{m_{1}, m_{2}, \ldots, m_{R}}=\gamma \prod_{r=1}^{R} e^{j \omega_{r} m_{r}}, \quad m_{r}=1,2, \ldots, M_{r}, \quad r=1,2, \ldots, R,
$$

The $\mathcal{Y} \in \mathbb{C}^{M_{1} \times M_{2} \times \cdots \times M_{R}}$ is the tensorial structured data set with length $M_{r}$ along the $r$ th dimension. The tensor $\mathcal{X}$ is the signal component where $\gamma$ and $\omega_{r} \in(-\pi, \pi)$ represent the unknown complex amplitude and frequency in the $r$ th dimension. On the other hand, the entries in $\mathcal{Q}$ are zero-mean complex white Gaussian noises with unknown variances $\sigma_{Q}^{2}$. Now the task is to find $\left\{\omega_{r}\right\}$ from the $M=\prod_{r=1}^{R} M_{r}$ samples of $\mathcal{Y}$.

By writing $\mathbf{g}_{r}=\left[\begin{array}{llll}e^{j \omega_{r}} & e^{j \omega_{r} 2} & \cdots & e^{j \omega_{r} M_{r}}\end{array}\right]^{T}$, to align with the presentation in Section 2 we define the $r$ th unfolding of $\mathcal{X}$ as the transpose version of $[19]$ :

$$
\begin{aligned}
{[\boldsymbol{\mathcal { X }}]_{(r)} } & =\gamma\left(\mathbf{g}_{r+1} \odot \cdots \odot \mathbf{g}_{R} \odot \mathbf{g}_{1} \odot \mathbf{g}_{2} \odot \cdots \mathbf{g}_{r-1}\right) \mathbf{g}_{r}^{T} \\
& =\left[\begin{array}{llll}
\mathbf{x}_{(r) 1} & \mathbf{x}_{(r) 2} & \cdots & \mathbf{x}_{(r) M_{r}}
\end{array}\right]
\end{aligned}
$$

and multiplying $[\boldsymbol{\mathcal { X }}]_{(r)}$ by $\mathbf{x}_{(r) 1}^{H}$ :

$$
\mathbf{c}^{(r)}=\mathbf{x}_{(r) 1}^{H}[\mathcal{X}]_{(r)}=\frac{M}{M_{r}}|\gamma|^{2} e^{-j \omega_{r}} \mathbf{g}_{r}^{T}
$$


Analogous to $(7)-(9)$, we define $[\mathcal{Y}]_{(r)}=\left[\begin{array}{llll}\mathbf{y}_{(r) 1} & \mathbf{y}_{(r) 2} & \cdots & \mathbf{y}_{(r) M_{r}}\end{array}\right]$ and $[\mathcal{Q}]_{(r)}=$ $\left[\begin{array}{llll}\mathbf{q}_{(r) 1} & \mathbf{q}_{(r) 2} & \cdots & \mathbf{q}_{(r) M_{r}}\end{array}\right]$ and construct

$$
\mathbf{z}_{(r)}^{T}=\mathbf{y}_{(r) 1}^{H}[\mathcal{Y}]_{(r)}
$$

where

$$
\left[\mathbf{z}_{(r)}\right]_{m_{r}}=e^{j \omega_{2}}\left[\mathbf{z}_{(r)}\right]_{m_{r}-1}+\mathbf{y}_{(r) 1}^{H}\left(\mathbf{q}_{(r) m_{r}}-e^{j \omega_{2}} \mathbf{q}_{(r) m_{r}-1}\right), \quad m_{r}=2,3, \cdots, M_{r}
$$

Generalizing (11), the WLS cost function is:

$$
J\left(\tilde{\rho}_{r}\right)=\left(\mathbf{z}_{(r) 2}-\tilde{\rho}_{r} \mathbf{z}_{(r) 1}\right)^{H} \mathbf{W}_{M_{r}-1}\left(\mathbf{z}_{(r) 2}-\tilde{\rho}_{r} \mathbf{z}_{(r) 1}\right)
$$

where $\tilde{\rho}_{r}=e^{-j \tilde{\omega}_{r}}$ while $\left.\mathbf{z}_{(r) 1}=\left[\begin{array}{llll}{\left[\mathbf{z}_{(r)}\right]_{1}} & {\left[\mathbf{z}_{(r)}\right]_{2}} & \cdots & {\left[\mathbf{z}_{(r)}\right.}\end{array}\right]_{M_{r}-1}\right]^{T}$ and $\mathbf{z}_{(r) 2}=$ $\left.\left[\begin{array}{llll}{\left[\mathbf{z}_{(r)}\right]_{2}} & {\left[\mathbf{z}_{(r)}\right]_{3}} & \cdots & {\left[\mathbf{z}_{(r)}\right.}\end{array}\right]_{M_{r}}\right]^{T}$. Following (10)-(15) and Appendix A with the assumption of SNR $>>M / M_{r}$, the C-1 estimate of $\omega_{r}, r=1,2, \cdots, R$, is:

$$
\hat{\omega}_{r}=\angle\left(\mathbf{z}_{(r) 1}^{H} \mathbf{W}_{M_{r}-1}\left(\omega_{r}\right) \mathbf{z}_{(r) 2}\right)
$$

Similarly, by discarding the first element in $\mathbf{z}_{(r)}, r=1,2, \cdots, R$, the C-2 frequency estimate has the same form as in (14). In Appendix B, we have proved that the higher dimensional C-1 and C-2 estimates are asymptotically unbiased and their variances equal (18) and (24), respectively. In summary, according to the development, the $\mathrm{C}-1$ estimator is more preferable when SNR $>>M / M_{r}$ while the C-2 method is a better choice if $M_{r}>>1, r=$ $1,2, \cdots, R$.

Finally, the complexity orders of the proposed methods are investigated as follows. In our study, the iterative procedure stops when the number of 
iterations reaches the value of $\tau$. According to Table 1, the major computations in the $r$ th dimension has a complexity of $\mathcal{O}\left(\tau M_{r}\right)$, implying that the orders of complexity for the $\mathrm{C}-1$ and C-2 algorithms are $\mathcal{O}\left(\sum_{r=1}^{R} \tau M_{r}\right)$ and $\mathcal{O}\left(\sum_{r=1}^{R} \tau\left(M_{r}-1\right)\right)$, respectively. As a comparison, the complexity order of the AIQML [15] method is $\mathcal{O}\left(\prod_{r=1}^{R} \tau M_{r}\right)$ while the IMDF [16] and UE [8] schemes have complexities of $\mathcal{O}\left(4 k_{t}\left(L^{E}-L^{E} / L_{1}\right) K^{E}\right)$ and $\mathcal{O}\left(2 k_{t} L^{E} K^{E}\right)$, respectively, where $k_{t}$ is a constant depends on the design of the SVD algorithm, $L_{r}^{E}+K_{r}^{E}-1=M_{r}, L_{r}^{E}>1, K_{r}^{E}>1, L^{E}=\prod_{r=1}^{R} L_{r}^{E}$ and $K^{E}=\prod_{r=1}^{R} K_{r}^{E}$. It can be roughly seen that when $\tau$ is small, the correlation-based methods are more computationally efficient.

\section{Numerical Examples}

Computer simulations have been conducted to evaluate the $R$-D singetone frequency estimation performance of the correlation-based approach in terms of mean square frequency error (MSFE) and complexity performance. We also include comparisons with the AIQML [15], IMDF [16] and UE [8] algorithms as well as CRLB [20]. Three iterations, that is, $\tau=3$, are employed as the stopping criterion in the proposed and AIQML methods because no significant improvement is observed for more iterations. The tone magnitude is $|\gamma|=1$ and its phase varies from $-\pi$ to $\pi$ in each independent trial. We properly scale the zero-mean white Gaussian noise data to produce different SNR conditions, where $\mathrm{SNR}=|\gamma|^{2} / \sigma_{Q}^{2}=1 / \sigma^{2}$, and all results provided are averages of 1000 independent.

In the first test, we solely compare the performance of the C-1 and C-2 methods. Figures 1 and 2 show the MSFEs versus SNR for the 2-D and 3-D 
cases with $\left[\begin{array}{ll}\omega_{1} & \omega_{2}\end{array}\right]=\left[\begin{array}{ll}0.3 & 0.05\end{array}\right] \pi$ and $\left[\begin{array}{lll}\omega_{1} & \omega_{2} & \omega_{3}\end{array}\right]=\left[\begin{array}{lll}0.3 & 0.05 & 0.9\end{array}\right] \pi$, respectively. The derived variances and CRLB are also included to verify the theoretical findings and investigate the algorithm optimality. Three choices of $N$, namely, $N=5, N=10$ and $N=20$, are studied where $M_{r}=N$ for all dimensions. We see from both figures that the performance of the C-1 and C-2 estimators agrees with the analytic expressions of (18) and (24), respectively, when $\mathrm{SNR} \geq 4 \mathrm{~dB}$. Although the C-1 method is generally superior to the C-2 algorithm, it is observed that the latter can give smaller $\mathrm{MSFE}$ at $\mathrm{SNR}=-10 \mathrm{~dB}$ when $N=20$ at $R=3$. Furthermore, comparing to the situation when the data length is smaller, namely, $\mathrm{N}=5$, the performance of the C-2 method is close to the CRLB for a larger $\mathrm{N}$, say $\mathrm{N}=20$, which also align with the development in Section 2.

In the second test, comparison is made with the AIQML and IMDF methods in 2-D estimation with $M_{1}=M_{2}=10$. The frequency parameters are $\left[\begin{array}{ll}\omega_{1} & \omega_{2}\end{array}\right]=\left[\begin{array}{ll}0.3 & 0.05\end{array}\right] \pi$ and the results are shown in Figure 3. We observe that the IMDF method has the best threshold performance at $\mathrm{SNR}=-6 \mathrm{~dB}$, while the AIQML and proposed methods have threshold SNRs at $-2 \mathrm{~dB}$ and OdB, respectively. Moreover, the MSFEs of the AIQML and C-1 schemes attain the CRLB at $\mathrm{SNR} \geq 10 \mathrm{~dB}$. On the other hand, the average computational times of the AIQML, IMDF, C-1 and C-2 algorithms for a single run are $7.85 \times 10^{-2} \mathrm{~s}, 3.07 \times 10^{-3} \mathrm{~s}, 2.18 \times 10^{-4} \mathrm{~s}$ and $1.88 \times 10^{-4} \mathrm{~s}$, respectively, indicating the attractiveness of the proposed approach in terms of complexity. It is seen that the computational times also agree with the complexity order analysis in Section 3.

In the third test, a comparative study in 3-D estimation is made with 
the UE and IMDF algorithms. The results for $M_{1}=M_{2}=M_{3}=10$ and $\left[\begin{array}{lll}\omega_{1} & \omega_{2} & \omega_{3}\end{array}\right]=\left[\begin{array}{lll}0.3 & 0.05 & 0.9\end{array}\right] \pi$ are plotted in Figure 4. At $\mathrm{SNR} \leq-2 \mathrm{~dB}$, the UE and IMDF methods outperform the proposed estimators. However, when $\mathrm{SNR}>5 \mathrm{~dB}$, the MSFE of the $\mathrm{C}-1$ scheme achieves the CRLB. The average computational times for the UE, IMDF, C-1 and C-2 algorithms for a single run are $1.13 \times 10^{-1} \mathrm{~s}, 2.23 \times 10^{-1} \mathrm{~s}, 3.92 \times 10^{-4} \mathrm{~s}$ and $3.14 \times 10^{-4} \mathrm{~s}$, which also agree with the complexity study in Section 3.

Finally, we perform comparison for different $R \in[3,5]$ with $M_{r}=4$, $r=1,2, \cdots, R$, and the results are shown in Figure 5 . The SNR is set to $20 \mathrm{~dB}$ and all the frequencies varies from $-0.99 \pi$ to $0.99 \pi$ in each independent run. It is seen that the C-1 algorithm performs the best and the theoretical calculations of (18) and (24) are again validated. Their average single run computational times are tabulated in Table 2, which agree with Section 3 and indicate the computational advantage of the proposed methodology particularly when the data dimension is large.

\begin{tabular}{|c|c|c|c|c|}
\hline$R$ & $\mathrm{UE}$ & $\mathrm{IMDF}$ & $\mathrm{C}-1$ & $\mathrm{C}-2$ \\
\hline 3 & $1.74 \times 10^{-3}$ & $1.47 \times 10^{-3}$ & $3.59 \times 10^{-4}$ & $3.06 \times 10^{-4}$ \\
\hline 4 & $4.68 \times 10^{-3}$ & $4.88 \times 10^{-3}$ & $4.46 \times 10^{-4}$ & $4.43 \times 10^{-4}$ \\
\hline 5 & $4.38 \times 10^{-2}$ & $4.02 \times 10^{-2}$ & $6.85 \times 10^{-4}$ & $5.94 \times 10^{-4}$ \\
\hline \hline
\end{tabular}

Table 2: Average computational times versus $R$

\section{Conclusion}

A fast and accurate multi-dimensional single frequency estimation approach based on correlation has been devised. Two estimators, C-1 and C-2, 
which are ideal for high SNR and/or large sample conditions, are proposed. The main idea in the algorithm development is to construct single-tone sequences which contain the frequency parameters by exploiting correlation of the observed data. Frequency estimation for each dimension is performed separately by using LP and WLS techniques. It is proved that the variances of the C-1 and C-2 estimators attain CRLB in the presence of white Gaussian noise, when the SNR and/or data length tend to infinity. Furthermore, the proposed correlation approach is more computationally attractive than the AIQML, UE and IMDF schemes, and provides superior estimation accuracy at higher SNRs. As a future work, we will extend the correlation-based methodology to estimation of multiple tones.

\section{Appendix A}

We now derive the weighting matrix for the general signal model of (26). According to (31), the LP residue error vector for the $r$ th dimension is

$$
\mathbf{e}_{(r)}=\mathbf{z}_{(r) 1}-\rho_{r} \mathbf{z}_{(r) 2}
$$

where $\left[\mathbf{e}_{(r)}\right]_{n_{r}}=\mathbf{y}_{(r) 1}^{H} \boldsymbol{\xi}_{(r) n_{r}}, n_{r}=1,2, \cdots, M_{r}-1, \boldsymbol{\xi}_{(r) n_{r}}=\left(\mathbf{q}_{(r)\left(n_{r}+1\right)}-\rho_{r} \mathbf{q}_{(r) n_{r}}\right)$ and $\rho_{r}=e^{j \omega_{r}}$. Applying the Gauss-Markov theorem [22], the optimal weighting matrix which is a function of $\rho_{r}$, denoted by $\mathbf{W}_{M_{r}-1}\left(\rho_{r}\right)$, is:

$$
\mathbf{W}_{M_{r}-1}\left(\rho_{r}\right)=\left[\mathbb{E}\left\{\mathbf{e}_{(r)} \mathbf{e}_{(r)}^{H}\right\}\right]^{-1}=\mathbf{B}^{-1}\left(\rho_{r}\right)
$$

where

$$
\begin{aligned}
{\left[\mathbf{B}\left(\rho_{r}\right)\right]_{m, n} } & =\mathbb{E}\left\{\left[\mathbf{e}_{(r)}\right]_{m}\left[\mathbf{e}_{(r)}\right]_{n}^{*}\right\}=\mathbb{E}\left\{\mathbf{y}_{(r) 1}{ }^{H} \boldsymbol{\xi}_{(r) m} \boldsymbol{\xi}_{(r) n}{ }^{H} \mathbf{y}_{(r) 1}\right\} \\
& =\left[\mathbf{K}_{1}\right]_{m, n}+\left[\mathbf{K}_{2}\right]_{m, n}+\left[\mathbf{K}_{3}\right]_{m, n}+\left[\mathbf{K}_{4}\right]_{m, n}, \quad m, n=1,2, \cdots, M_{r}-1
\end{aligned}
$$


with

$$
\begin{aligned}
{\left[\mathbf{K}_{1}\right]_{m, n} } & =\mathbb{E}\left\{\mathbf{x}_{(r) 1}^{H} \boldsymbol{\xi}_{(r) m} \boldsymbol{\xi}_{(r) n}^{H} \mathbf{x}_{(r) 1}\right\} \\
{\left[\mathbf{K}_{2}\right]_{m, n} } & =\mathbb{E}\left\{\mathbf{q}_{(r) 1}^{H} \boldsymbol{\xi}_{(r) m} \boldsymbol{\xi}_{(r) n}^{H} \mathbf{q}_{(r) 1}\right\} \\
{\left[\mathbf{K}_{3}\right]_{m, n} } & =\mathbb{E}\left\{\mathbf{x}_{(r) 1}^{H} \boldsymbol{\xi}_{(r) m} \boldsymbol{\xi}_{(r) n}^{H} \mathbf{q}_{(r) 1}\right\} \\
{\left[\mathbf{K}_{4}\right]_{m, n} } & =\mathbb{E}\left\{\mathbf{q}_{(r) 1}^{H} \boldsymbol{\xi}_{(r) m} \boldsymbol{\xi}_{(r) n}^{H} \mathbf{x}_{(r) 1}\right\}
\end{aligned}
$$

On the other hand, since $\mathcal{Q}$ contains zero-mean independent and identically distributed Gaussian samples, we get:

$$
\mathbb{E}\left\{\mathbf{x}_{(r) 1}^{H} \mathbf{q}_{(r) p}\right\}=\mathbb{E}\left\{\mathbf{q}_{(r) p}^{H} \mathbf{q}_{(r) q}\right\}=0
$$

and

$$
\mathbb{E}\left\{\mathbf{q}_{(r) p}^{H} \mathbf{q}_{(r) p}\right\}=\frac{M}{M_{r}} \sigma_{Q}^{2}
$$

for any $p \neq q, p, q=1,2, \cdots, M_{r}$, which result in $\mathbf{K}_{3}=\mathbf{K}_{4}=\mathbf{0}_{\left(M_{r}-1\right) \times\left(M_{r}-1\right)}$ and

$$
\mathbb{E}\left\{\boldsymbol{\xi}_{(r) m} \boldsymbol{\xi}_{(r) n}^{H}\right\}= \begin{cases}\sigma_{Q}^{2}\left(1+\left|\rho_{r}\right|^{2}\right) \mathbf{I}_{\frac{M}{M_{r}}}, & m=n \\ -\sigma_{Q}^{2} \rho_{r}^{*} \mathbf{I}_{\frac{M}{M_{r}}}, & m=n+1 \\ -\sigma_{Q}^{2} \rho_{r} \mathbf{I}_{\frac{M}{M_{r}}}, & m=n-1 \\ 0, & \text { otherwise }\end{cases}
$$

Therefore

$$
\mathbf{K}_{1}=\frac{M}{M_{r}}|\gamma|^{2} \sigma_{Q}^{2}\left[\begin{array}{cccccc}
\left(1+\left|\rho_{r}\right|^{2}\right) & -\rho_{r} & 0 & 0 & \ldots & 0 \\
-\rho_{r}^{*} & \left(1+\left|\rho_{r}\right|^{2}\right) & -\rho_{r} & 0 & \ldots & 0 \\
\vdots & \vdots & \vdots & \vdots & \vdots & \vdots \\
0 & \ldots & 0 & -\rho_{r}^{*} & \left(1+\left|\rho_{r}\right|^{2}\right) & -\rho \\
0 & 0 & \ldots & 0 & -\rho_{r}^{*} & \left(1+\left|\rho_{r}\right|^{2}\right)
\end{array}\right]
$$


To calculate $\mathbf{K}_{2}$, we notice that when $p \neq 1, \mathbf{q}_{(r) 1}$ and $\boldsymbol{\xi}_{(r) p}$ are independent to each other, meaning that $\mathbb{E}\left\{\mathbf{q}_{(r) 1}^{H} \boldsymbol{\xi}_{(r) p}\right\}=0$. Hence $\left[\mathbf{K}_{2}\right]_{m, n}=\left[\mathbf{K}_{1}\right]_{m, n} \sigma_{Q}^{2} /|\gamma|^{2}$ for any $m \neq 1$ or $n \neq 1$, and $\left[\mathbf{K}_{2}\right]_{1,1}=\mathbb{E}\left\{\mathbf{q}_{(r) 1}^{H} \boldsymbol{\xi}_{(r) 1} \boldsymbol{\xi}_{(r) 1}^{H} \mathbf{q}_{(r) 1}\right\}=M / M_{r}(1+$ $\left.M / M_{r}\left|\rho_{r}\right|^{2}\right) \sigma_{Q}^{4}$. Substituting $\mathbf{K}_{1}, \mathbf{K}_{2}$ and $\rho_{r}=e^{j \omega_{r}}$ into (A.3) yields

$$
\mathbf{B}\left(\omega_{r}\right)=\frac{M}{M_{r}}\left(|\gamma|^{2}+\sigma_{Q}^{2}\right) \sigma_{Q}^{2} \mathbf{P}\left(\omega_{r}\right)
$$

where

$$
\mathbf{P}\left(\omega_{r}\right)=\left[\begin{array}{cccccc}
2+\left(M / M_{r}-1\right) \sigma_{Q}^{2} /\left(|\gamma|^{2}+\sigma_{Q}^{2}\right) & -e^{j \omega_{r}} & 0 & 0 & \cdots & 0 \\
-e^{-j \omega_{r}} & 2 & -e^{j \omega_{r}} & 0 & \cdots & 0 \\
\vdots & \vdots & \vdots & \vdots & \vdots & \vdots \\
0 & \cdots & 0 & -e^{-j \omega_{r}} & 2 & -e^{j \omega_{r}} \\
0 & 0 & \cdots & 0 & -e^{-j \omega_{r}} & 2
\end{array}\right]
$$

As $M / M_{r}\left(|\gamma|^{2}+\sigma_{Q}^{2}\right) \sigma_{Q}^{2}$ is just a scalar, we can simply write $\mathbf{W}_{M_{r}-1}\left(\rho_{r}\right)=$ $\mathbf{W}_{M_{r}-1}\left(\omega_{r}\right)=\mathbf{P}^{-1}\left(\omega_{r}\right)$. Note that $(7)-(8)$ correspond to the special case of 2-D estimation.

\section{Appendix B}

The bias and variance of $\hat{\omega}_{r}, r=1,2, \cdots, R$, for the general model of (26) are now analyzed. Let the bias of $\hat{\omega}_{r}$ be $\Delta \omega_{r}=\hat{\omega}_{r}-\omega_{r}$. Following [18], for sufficiently high SNR conditions, we have

$$
\begin{aligned}
\Delta \omega_{r} & \approx \frac{\kappa_{r}}{\overline{\mathbf{z}}_{(r) 1}^{H} \mathbf{W}_{M_{r}-1}\left(\omega_{r}\right) \overline{\mathbf{z}}_{(r) 1}} \\
\operatorname{var}\left(\hat{\omega}_{r}\right) & \approx \frac{\mathbb{E}\left\{\kappa_{r}^{2}\right\}}{\left(\mathbb{E}\left\{\overline{\mathbf{z}}_{(r) 1}^{H} \mathbf{W}_{M_{r}-1}\left(\omega_{r}\right) \overline{\mathbf{z}}_{(r) 1}\right\}\right)^{2}}
\end{aligned}
$$


where

$$
\kappa_{r} \approx \Im\left\{e^{-j \omega_{r}}\left(\overline{\mathbf{z}}_{(r) 1}^{H} \mathbf{W}_{M_{r}-1}\left(\omega_{r}\right) \Delta \mathbf{z}_{(r) 2}+\Delta \mathbf{z}_{(r) 1}^{H} \mathbf{W}_{M_{r}-1}\left(\omega_{r}\right) \overline{\mathbf{z}}_{(r) 2}\right)\right\}
$$

with $\mathbf{z}_{(r) i}=\overline{\mathbf{z}}_{(r) i}+\Delta \mathbf{z}_{(r) i}, i=1,2$. Notice that $\overline{\mathbf{z}}_{(r)}^{T}=\left(\mathbf{x}_{(r) 1}+\mathbf{q}_{(r) 1}\right)^{H}$ $\left[\begin{array}{llll}\mathbf{x}_{(r) 1} & \mathbf{x}_{(r) 2} & \cdots & \mathbf{x}_{(r) M_{r}}\end{array}\right]$ and $\boldsymbol{\Delta} \mathbf{z}_{(r)}^{T}=\left(\mathbf{x}_{(r) 1}+\mathbf{q}_{(r) 1}\right)^{H}\left[\begin{array}{llll}\mathbf{q}_{(r) 1} & \mathbf{q}_{(r) 2} & \cdots & \mathbf{q}_{(r) M_{r}}\end{array}\right]$.

According to (A.8)-(A.9), we obtain

$$
\begin{aligned}
& \mathbb{E}\left\{\mathbf{x}_{(r)(m)}^{H}\left(\mathbf{x}_{(r) 1}+\mathbf{q}_{(r) 1}\right)\left(\mathbf{x}_{(r) 1}+\mathbf{q}_{(r) 1}\right)^{H} \mathbf{x}_{(r)(n)}\right\} \\
= & \left.\mathbf{x}_{(r)(m)}^{H} \mathbf{x}_{(r) 1} \mathbf{x}_{(r) 1}^{H} \mathbf{x}_{(r)(n)}+\mathbf{x}_{(r)(m)}^{H} \mathbb{E}\left\{\mathbf{q}_{(r) 1}\right)\left(\mathbf{q}_{(r) 1}\right)^{H}\right\} \mathbf{x}_{(r)(n)} \\
= & e^{-j(m-n)} \frac{M}{M_{r}}\left(\frac{M}{M_{r}}|\gamma|^{2}+\sigma_{Q}^{2}\right)|\gamma|^{2}
\end{aligned}
$$

and

$$
\mathbb{E}\left\{\Delta \mathbf{z}_{(r)}\right\}=\left[\begin{array}{lllll}
M / M_{r} \sigma_{Q}^{2} & 0 & 0 & \cdots & 0
\end{array}\right]^{T}
$$

Therefore the expected value of the denominator of (B.1) is:

$$
\begin{aligned}
& \mathbb{E}\left\{\overline{\mathbf{z}}_{(r) 1}^{H} \mathbf{W}_{M_{r}-1}\left(\omega_{r}\right) \overline{\mathbf{z}}_{(r) 1}\right\} \\
\approx & \sum_{m=1}^{M_{r}-1} \sum_{n=1}^{M_{r}-1} \mathbb{E}\left\{\mathbf{x}_{(r) m}^{H}\left(\mathbf{x}_{(r) 1}+\mathbf{q}_{(r) 1}\right)\left(M_{r} \min (m, n)-m n\right) e^{j(m-n) \omega}\left(\mathbf{x}_{(r) 1}+\mathbf{q}_{(r) 1}\right)^{H} \mathbf{x}_{(r) n}\right\} \\
= & \frac{M}{M_{r}^{2}}\left(\frac{M}{M_{r}}|\gamma|^{2}+\sigma_{Q}^{2}\right)|\gamma|^{2}\left(\sum_{m=1}^{M_{r}-1}\left(M_{r} m-m^{2}\right)+\sum_{m=1}^{M_{r}-1} \sum_{n=1}^{m-1}\left(M_{r} n-m n\right)+\right. \\
& \left.\sum_{n=1}^{M_{r}-1} \sum_{m=1}^{n-1}\left(M_{r} m-m n\right)\right) \\
= & \frac{M\left(M_{r}^{2}-1\right)}{12}\left(\frac{M}{M_{r}}|\gamma|^{2}+\sigma_{Q}^{2}\right)|\gamma|^{2}
\end{aligned}
$$

On the other hand, $\kappa_{r}$ can be written as:

$$
\kappa_{r}=t_{r 1}+t_{r 2}
$$




$$
\begin{aligned}
& \text { where } t_{r 1}=\frac{e^{-j \omega_{r}} \overline{\mathbf{z}}_{(r) 1}^{H} \mathbf{W}_{M_{r}-1}\left(\omega_{r}\right) \Delta \mathbf{z}_{(r) 2}-e^{j \omega_{r}} \overline{\mathbf{z}}_{(r) 2}^{H} \mathbf{W}_{M_{r}-1}\left(\omega_{r}\right) \Delta \mathbf{z}_{(r) 1}}{2 j} \\
& =\frac{\overline{\mathbf{z}}_{(r) 2}^{H} \mathbf{W}_{M_{r}-1}\left(\omega_{r}\right)\left(\Delta \mathbf{z}_{(r) 2}-\rho_{r} \Delta \mathbf{z}_{(r) 1}\right)}{2 j} \\
& t_{r 2}=\frac{e^{-j \omega_{r}} \overline{\mathbf{z}}_{(r) 2}^{T} \mathbf{W}_{M_{r}-1}^{*}\left(\omega_{r}\right) \Delta \mathbf{z}_{(r) 1}^{*}-e^{j \omega_{r}} \overline{\mathbf{z}}_{(r) 1}^{T} \mathbf{W}_{M_{r}-1}^{*}\left(\omega_{r}\right) \Delta \mathbf{z}_{(r) 2}^{*}}{2 j} \\
& =-\frac{\left(\Delta \mathbf{z}_{(r) 2}-\rho_{r} \boldsymbol{\Delta} \mathbf{z}_{(r) 1}\right)^{H} \mathbf{W}_{M_{r}-1}\left(\omega_{r}\right) \overline{\mathbf{z}}_{(r) 2}}{2 j}
\end{aligned}
$$

because $\overline{\mathbf{z}}_{(r) 2}=\rho_{r} \overline{\mathbf{z}}_{(r) 1}, \rho_{r}=e^{j \omega_{r}}$ and $\mathbf{W}_{M_{r}-1}\left(\omega_{r}\right)=\mathbf{W}_{M_{r}-1}^{H}\left(\omega_{r}\right)$. As $\mathbb{E}\left\{\boldsymbol{\Delta} \mathbf{z}_{(r) 2}\right\}=\mathbf{0}_{\left(M_{r}-1\right) \times 1}$, we have:

$$
\begin{aligned}
\mathbb{E}\left\{t_{r 1}\right\}= & -\frac{1}{2 j} \mathbb{E}\left\{\rho_{r} \overline{\mathbf{z}}_{(r) 2}^{H} \mathbf{W}_{M_{r}-1}\left(\omega_{r}\right) \Delta \mathbf{z}_{(r) 1}\right\}+0=-\frac{1}{2 j} \mathbb{E}\left\{\overline{\mathbf{z}}_{(r) 1}^{H} \mathbf{W}_{M_{r}-1}\left(\omega_{r}\right) \Delta \mathbf{z}_{(r) 1}\right\} \\
= & -\frac{1}{2 j} \sum_{m=1}^{M_{r}-1} \sum_{n=1}^{M_{r}-1} \mathbb{E}\left\{\mathbf{x}_{(r) m}^{H}\left(\mathbf{x}_{(r) 1}+\mathbf{q}_{(r) 1}\right)\left(M_{r} \min (m, n)-m n\right) e^{j(m-n) \omega}\right. \\
& \left.\left(\mathbf{x}_{(r) 1}+\mathbf{q}_{(r) 1}\right)^{H} \mathbf{q}_{(r) n}\right\} \\
= & -\frac{1}{2 j} \sum_{m=1}^{M_{r}-1} \mathbb{E}\left\{\mathbf{x}_{(r) m}^{H}\left(\mathbf{x}_{(r) 1}+\mathbf{q}_{(r) 1}\right)\left(M_{r}-m\right) e^{j(1-m) \omega}\left(\mathbf{x}_{(r) 1}+\mathbf{q}_{(r) 1}\right)^{H} \mathbf{q}_{(r) 1}\right\}+0 \\
= & -\frac{1}{2 j} \sum_{m=1}^{M_{r}-1}\left(M_{r}-m\right) \mathbb{E}\left\{\mathbf{x}_{(r) 1}^{H}\left(\mathbf{x}_{(r) 1}+\mathbf{q}_{(r) 1}\right)\left(\mathbf{x}_{(r) 1}+\mathbf{q}_{(r) 1}\right)^{H} \mathbf{q}_{(r) 1}\right\} \\
= & -\frac{1}{2 j} \sum_{n=1}^{M_{r}-1}\left(M_{r}-n\right) \mathbb{E}\left\{\mathbf{x}_{(r) 1}^{H} \mathbf{x}_{(r) 1} \mathbf{q}_{(r) 1}^{H} \mathbf{q}_{(r) 1}\right\}+0 \\
= & j \frac{M^{2}\left(M_{r}-1\right)}{4 M_{r}}|\gamma|^{2} \sigma_{Q}^{2}
\end{aligned}
$$

and $\mathbb{E}\left\{t_{r 2}\right\}=-\mathbb{E}\left\{t_{r 1}\right\}$. This means that $\mathbb{E}\left\{\Delta \omega_{r}\right\} \approx \mathbb{E}\left\{\kappa_{r}\right\}=0$ when SNR is sufficiently high, indicating the asymptotically unbiasedness of $\hat{\omega}_{r}$.

To calculate (B.2), we notice that

$$
\begin{aligned}
\mathbb{E}\left\{\kappa_{r}^{2}\right\} & =\mathbb{E}\left\{t_{r 1} t_{r 2}^{*}\right\}+\mathbb{E}\left\{t_{r 2} t_{r 1}^{*}\right\}+\mathbb{E}\left\{t_{r 1} t_{r 1}^{*}\right\}+\mathbb{E}\left\{t_{r 2} t_{r 2}^{*}\right\} \\
& =2 \mathbb{E}\left\{t_{r 1} t_{r 2}\right\}+\mathbb{E}\left\{t_{r 1}^{2}\right\}+\mathbb{E}\left\{t_{r 2}^{2}\right\}
\end{aligned}
$$


where

$$
\mathbb{E}\left\{t_{r 1}^{2}\right\}=\mathbb{E}\left\{t_{r 2}^{2}\right\}=-\frac{M^{4}\left(M_{r}-1\right)^{2}}{16 M_{r}^{2}}|\gamma|^{4} \sigma_{Q}^{4}
$$

and

$$
\begin{aligned}
\mathbb{E}\left\{t_{r 1} t_{r 2}\right\} & =\frac{1}{4} \mathbb{E}\left\{\overline{\mathbf{z}}_{(r) 2}^{H} \mathbf{W}_{M_{r}-1}\left(\omega_{r}\right)\left(\Delta \mathbf{z}_{(r) 2}-\rho_{r} \boldsymbol{\Delta} \mathbf{z}_{(r) 1}\right)\left(\Delta \mathbf{z}_{(r) 2}-\rho_{r} \Delta \mathbf{z}_{(r) 1}\right)^{H} \mathbf{W}_{M_{r}-1}\left(\omega_{r}\right) \overline{\mathbf{z}}_{(r) 2}\right\} \\
& \approx \frac{1}{4} \mathbb{E}\left\{\overline{\mathbf{z}}_{(r) 2}^{H} \mathbf{W}_{M_{r}-1}\left(\omega_{r}\right) \mathbf{B}\left(\omega_{r}\right) \mathbf{W}_{M_{r}-1}\left(\omega_{r}\right) \overline{\mathbf{z}}_{(r) 2}\right\} \\
& =\frac{1}{4} \mathbb{E}\left\{\overline{\mathbf{z}}_{(r) 2}^{H} \mathbf{W}_{M_{r}-1}\left(\omega_{r}\right) \frac{M}{M_{r}}\left(|\gamma|^{2}+\sigma_{Q}^{2}\right) \sigma_{Q}^{2} \mathbf{W}_{M_{r}-1}^{-1}\left(\omega_{r}\right) \mathbf{W}_{M_{r}-1}\left(\omega_{r}\right) \overline{\mathbf{z}}_{(r) 2}\right\} \\
& =\frac{M}{4 M_{r}}\left(|\gamma|^{2}+\sigma_{Q}^{2}\right) \sigma_{Q}^{2} \mathbb{E}\left\{\overline{\mathbf{z}}_{(r) 1}^{H} \mathbf{W}_{M_{r}-1}\left(\omega_{r}\right) \overline{\mathbf{z}}_{(r) 1}\right\}
\end{aligned}
$$

For sufficiently high SNRs, $\left|\mathbb{E}\left\{t_{r 1}^{2}\right\}\right| \ll \mathbb{E}\left\{t_{r 1} t_{r 2}\right\}$, and substituting (B.6) and (B.11)-(B.13) into (B.2), we obtain (18).

\section{Acknowledgement}

The work described in this paper was fully supported by a grant from the Research Grants Council of the Hong Kong Special Administrative Region, China [Project No. CityU 120911].

\section{References}

[1] S.L. Marple, Digital Spectral Analysis with Applications, Englewood Cliffs, NJ: Prentice-Hall, 1987

[2] S.M. Kay, Modern Spectral Estimation: Theory and Application, Englewood Cliffs, NJ: Prentice-Hall, 1988

[3] P. Stoica and R. Moses, Spectral Analysis of Signals, Upper Saddle River, NJ: Prentice-Hall, 2005 
[4] F.K.W. Chan, H.C. So, W.H. Lau and C.F. Chan, "Structured total least squares approach for efficient frequency estimation," Signal Processing, vol.91, no.4, pp.1043-1047, Apr. 2011

[5] K.W.K. Lui and H.C. So, "Two-stage autocorrelation approach for accurate single sinusoidal frequency estimation," Signal Processing, vol.88, no.7, pp.1852-1857, Jul. 2008

[6] Z. Zhou and H.C. So, "Linear prediction approach to oversampling parameter estimation for multiple complex sinusoids", Signal Processing, vol.92, no.6, pp.1458-1466, Jun. 2012

[7] J.H. McClellan, "Multidimensional spectral estimation," Proceedings of the IEEE, vol.70, no.9, pp.1029-1039, Sep. 1982

[8] M. Haardt and J.A. Nossek, "Simultaneous Schur decomposition of several nonsymmetric matrices to achieve automatic pairing in multidimensional harmonic retrieval problems," IEEE Transactions on Signal Processing, vol.40, no.1, pp.161-169, Jan. 1998

[9] H.L. van Trees, Optimum Array Processing: Detection, Estimation, and Modulation Theory. New York: Wiley, 2002, pt. IV

[10] Y. Li, J. Razavilar and K.J.R. Liu, "A high-resolution technique for multidimensional NMR spectroscopy," IEEE Transactions on Biomedical Engineering, vol.45, pp.78-86, Jan. 1998

[11] K.N. Mokios, N.D. Sidiropoulos, M. Pesavento and C.F. Mecklenbrauker, "On 3-D harmonic retrieval for wireless channel sounding," 
Proc., IEEE International Conference on Acoustics, Speech and Signal Processing, vol. 2, pp. 1189-1192, Montreal, Quebec, Canada, May 2004

[12] M. Pesavento, C.F. Mecklenbraker and J.F. Bome, "Multi-dimensional rank reduction estimator for parametric MIMO channel models," EURASIP J. Appl. Signal Process., vol. 2004, pp. 1354-363, Sep. 2004

[13] D. Nion and N.D. Sidiropoulos, "Tensor algebra and multidimensional harmonic retrieval in signal processing for MIMO radar" IEEE Transactions on Signal Processing, vol.58, no.11, pp.5693-5705, Nov. 2010

[14] M.P. Clark and L.L. Scharf, "Two-dimensional modal analysis based on maximum likelihood," IEEE Transactions on Signal Processing, vol.42, no.6, pp.1443-1452, Jun. 1994

[15] H.C. So and F.K.W. Chan, "Approximate maximum-likelihood algorithms for two-dimensional frequency estimation of a complex sinusoid," IEEE Transactions on Signal Processing, vol.54, no.8, pp.3231-3237, Aug. 2006

[16] J. Liu and X. Liu, "An eigenvector-based approach for multidimensional frequency estimation with improved identifiability," IEEE Transactions on Signal Processing, vol.54, no.12, pp.4543-4556, Dec. 2006

[17] R. Boyer, "Decoupled root-music algorithm for multidimensional harmonic retrieval," IEEE Workshop on Signal Processing Advances in Wireless Communications, pp. 16-20, Recife, Brazil, Jul. 2008 
[18] H.C. So and F.K.W. Chan, "A generalized weighted linear predictor frequency estimation approach for a complex sinusoid," IEEE Transactions on Signal Processing, vol.54, no.4, pp. 1304-1315, Apr. 2006

[19] L. de Lathauwer, B. de Moor, and J. Vanderwalle, "A multilinear singular value decomposition," SIAM J. Matrix Anal. Appl., vol. 21, no. 4, pp. $1253-1278,2000$

[20] R. Boyer, "Deterministic asymptotic Cramer-Rao lower bound for the multidimensional harmonic model," Signal Processing, vol. 88, no. 12, pp. 2869-2877, Dec. 2008

[21] S. Kay, "A fast and accurate single frequency estimator," IEEE Trans. Acoust., Speech, Signal Processing, vol.37, pp.1987-1990, Dec. 1989

[22] S.M. Kay, Fundamentals of Statistical Signal Processing: Estimation Theory, Englewood Cliffs, NJ: Prentice Hall, 1993 


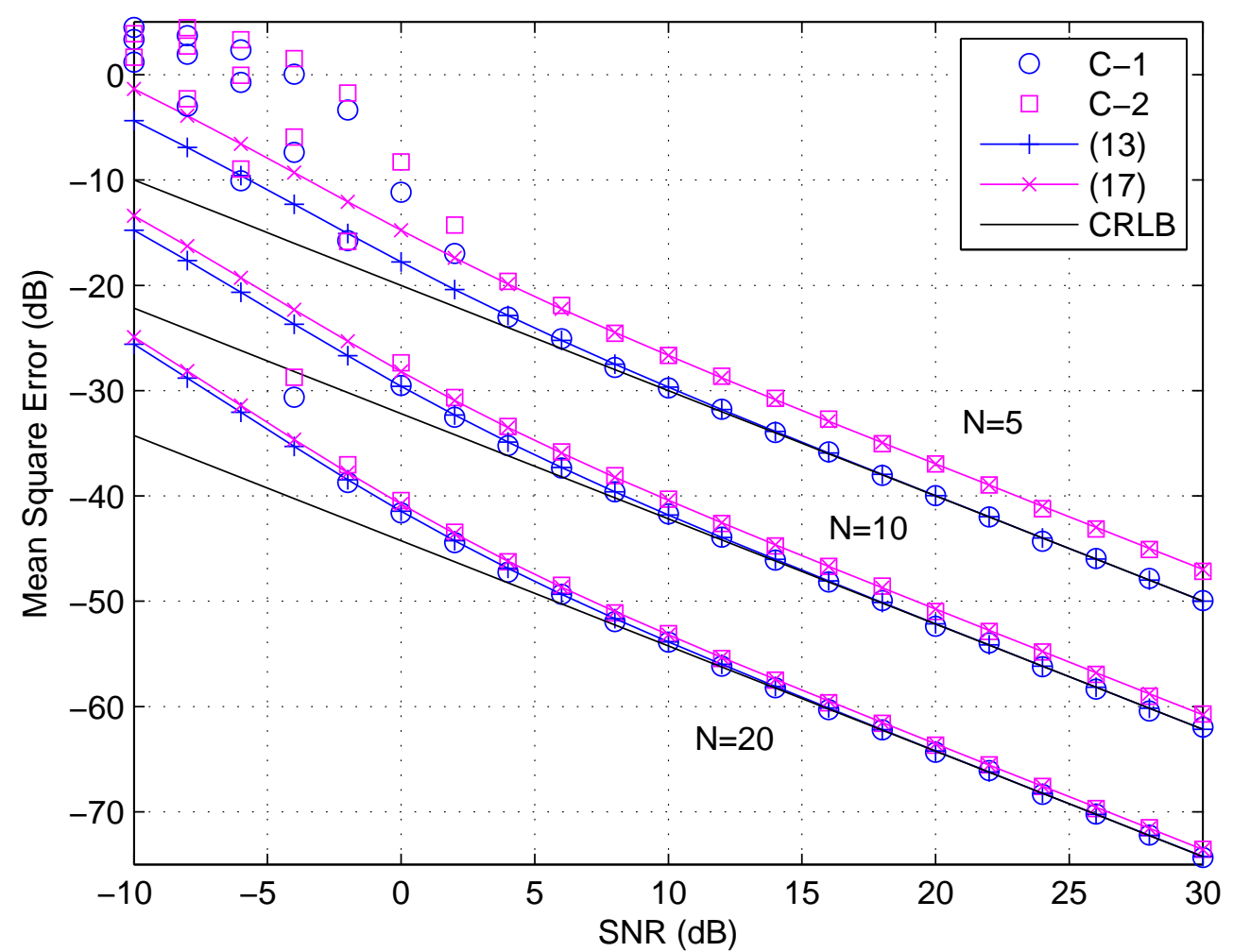

Figure 1: Average mean square frequency error versus SNR at $M_{1}=M_{2}=N$ 


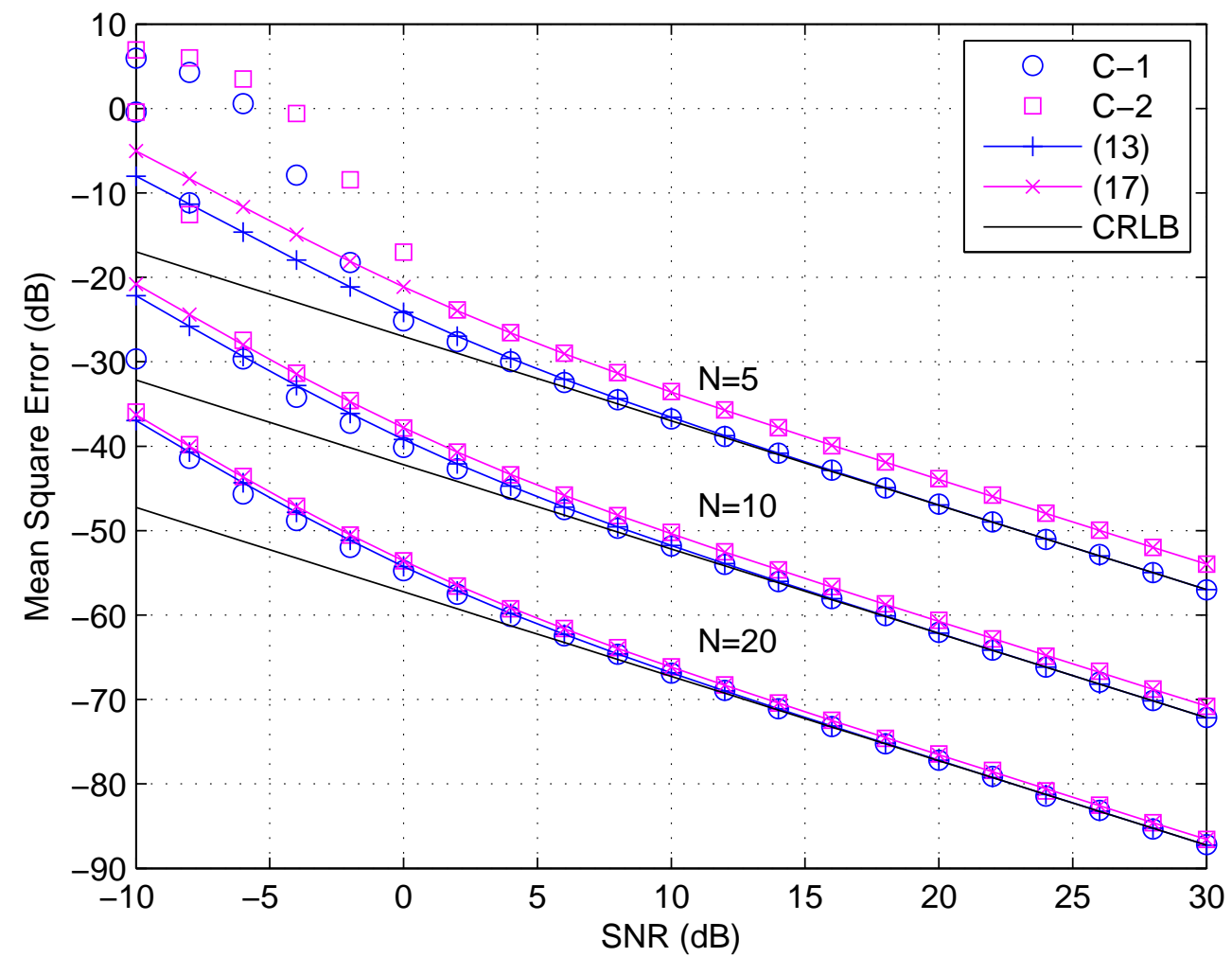

Figure 2: Average mean square frequency error versus SNR at $M_{1}=M_{2}=M_{3}=N$ 


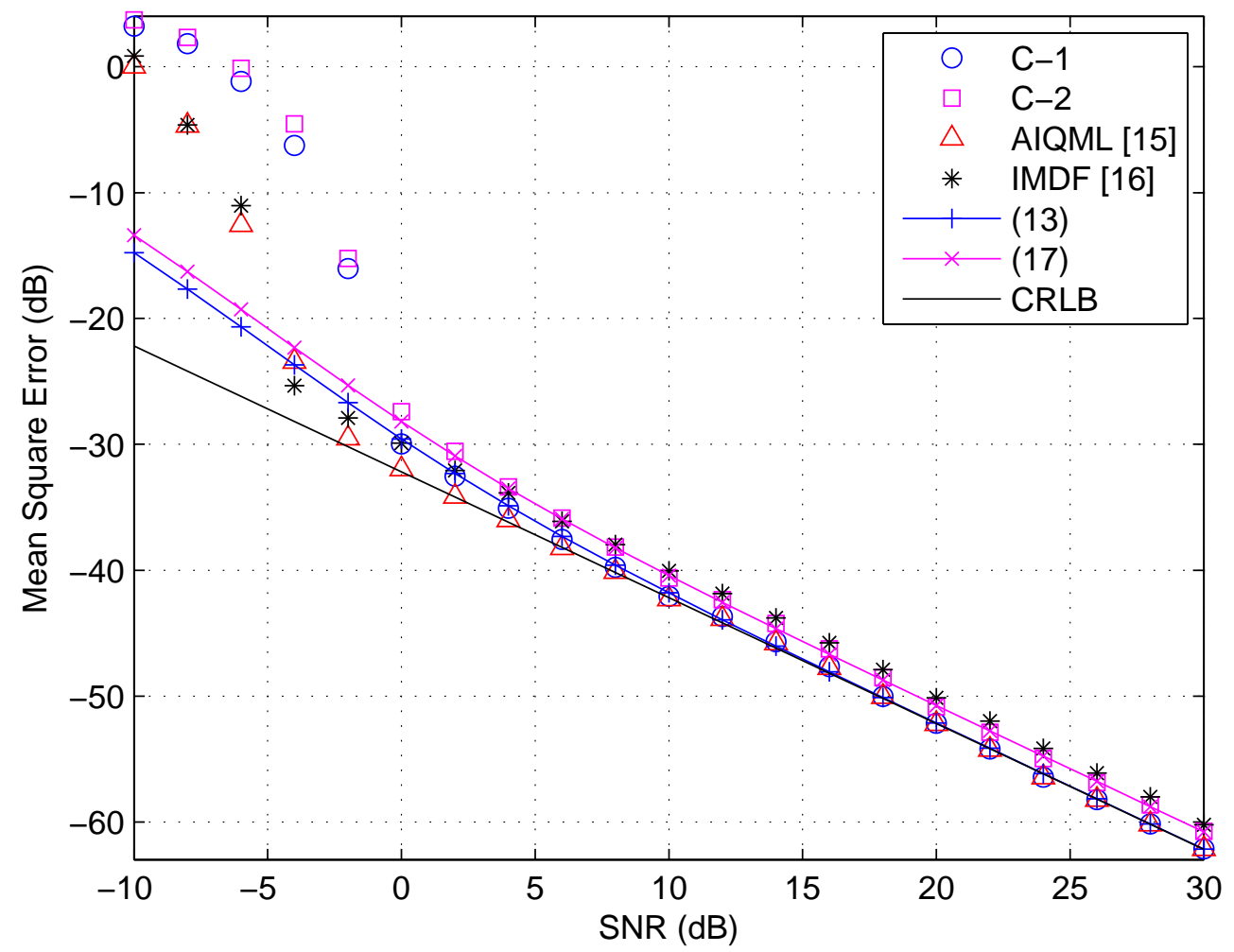

Figure 3: Average mean square frequency error versus SNR for 2-D case 


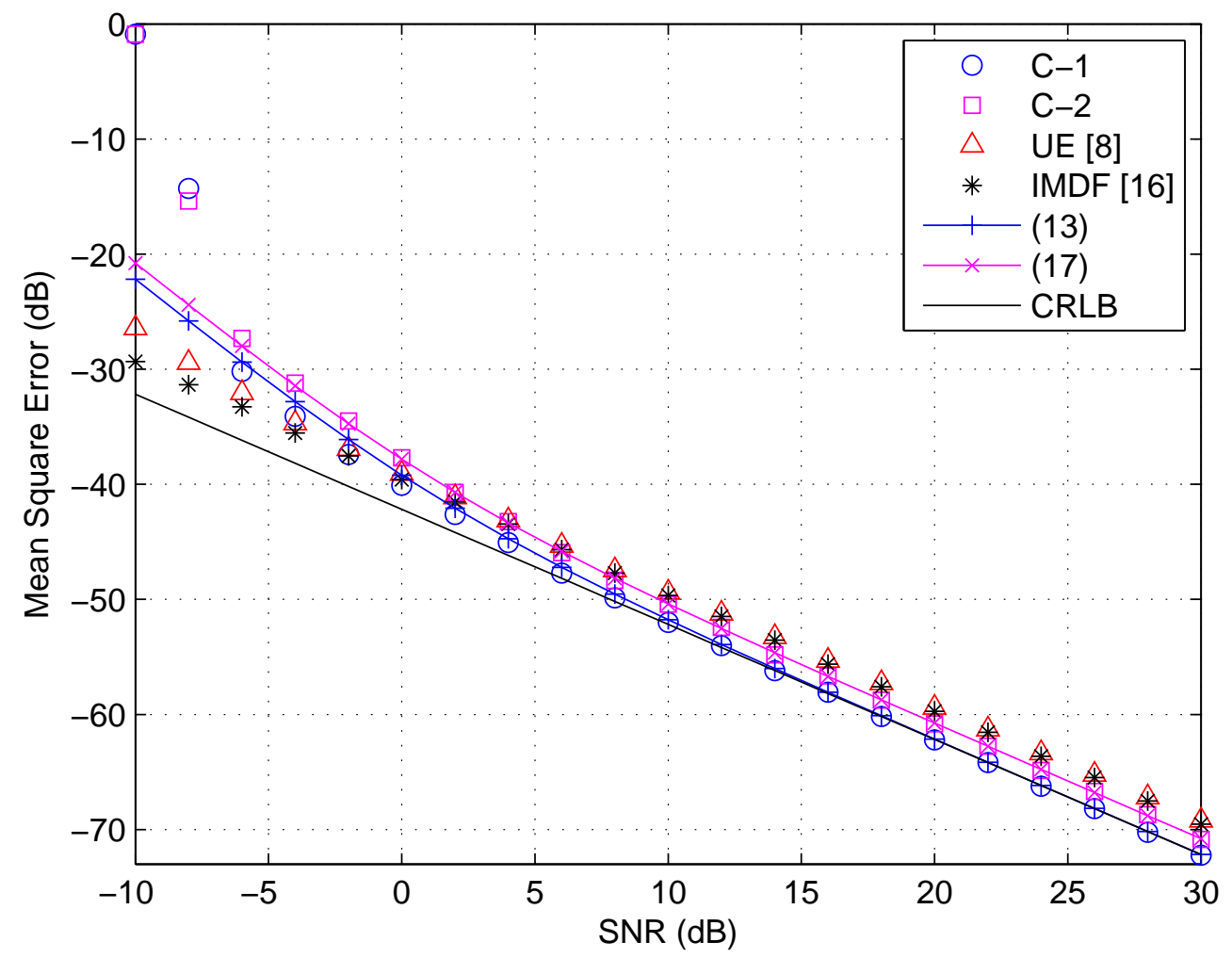

Figure 4: Average mean square frequency error versus SNR for 3-D case 


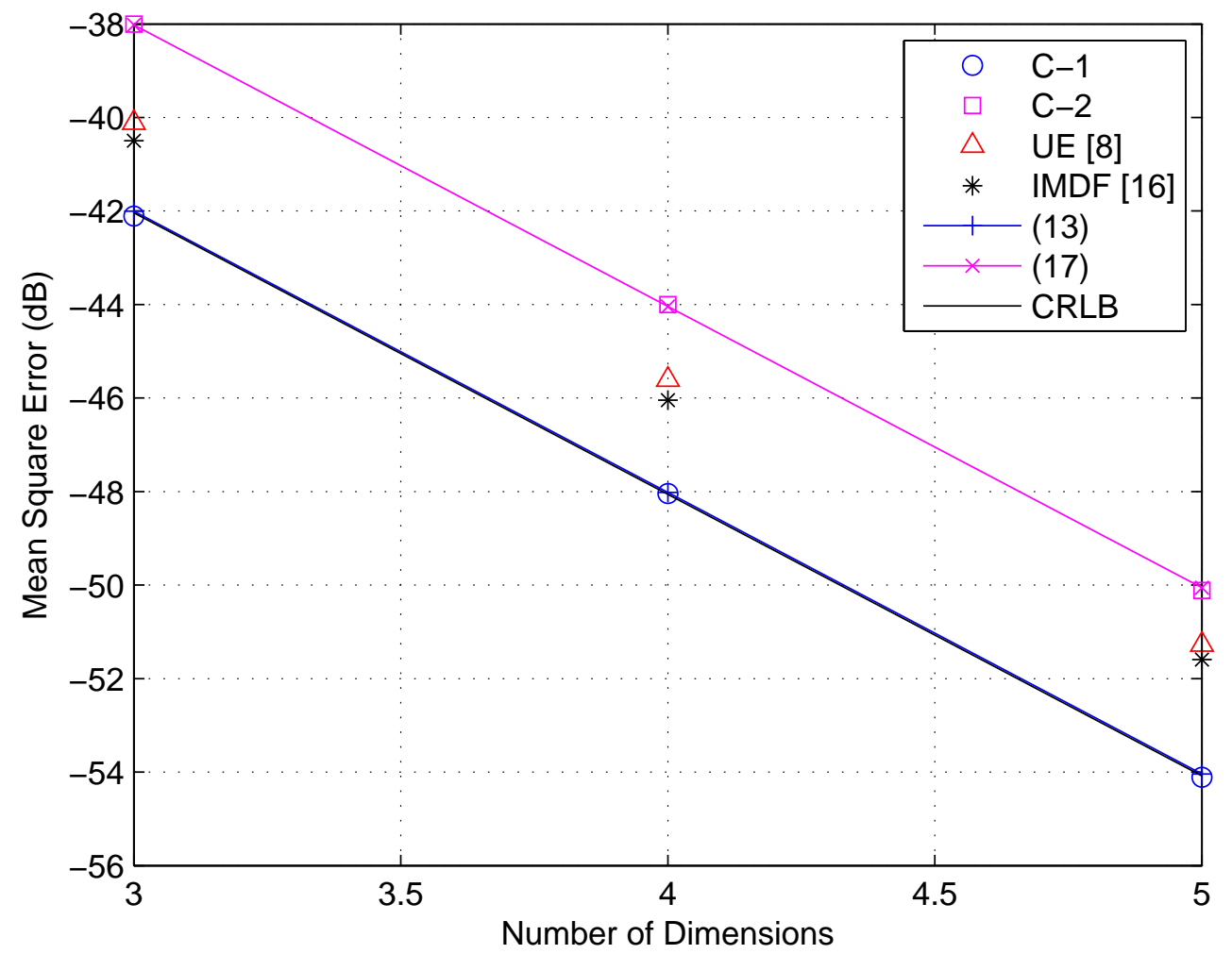

Figure 5: Average mean square frequency error versus $R$ at $M_{r}=4$ and $\mathrm{SNR}=20 \mathrm{~dB}$ 